\title{
Response to Response
}

\section{Soleve Image-Guided Targeted Hyperstimulation Analgesia Show Promising Clinical Results in Chronic Low Back Pain}

The reanalysis presented in our letter to the editor has been executed, reported and sent to the Company by Nijs group. In fact, the graphical presentation, the results and the conclusion are quoted word for word from the report by Nijs group. This reanalysis was performed by Nijs group following a detailed face to face discussion of the findings.

Nijs found promising clinical results with the new device, which seems to reduce self-reported pain by approximately $50 \%$ in the experimental group, compared to the placebo control-group, thereby contradicting the published data in Pain Physician journal by the same author (1).
To avoid any doubt it is quoted here again:

Results

VAS pain: A significant Time $x$ Group interaction was found for VAS-pain $(F(5,117)=2.708, p<.05)$. This interaction is presented in Figure 2. Following this interaction, a simple-effects analysis revealed that pain levels of patients in the experimental group were significantly lower before session 5 ( $p$ $=.05)$ but not lower before session $6(p>.05)$. Conclusions: We must be very cautious due to the small number of patients in this reanalysis. Looking at pain levels, the new device seems to reduce 
self-reported pain by approximately $50 \%$ in the experimental group, compared to the placebo treatment control group. Further research is required with a larger sample and with patients having either a placebo or experimental pain reduction (via the device) but only when the device is adequately functioning in its pain localization.

As the Company had no access to patient evaluation data during the entire course of the RCT, nor during the classification of scans and not until today, the results could not have been anticipated or influenced. Moreover, from a scientific stand point, these results indicate that there is significant correlation between the diagnostic information, i.e. the scans, and patient response to treatment. Therefore are clinically meaningful and must not be avoided.

We could not understand the real motivation of Nijs in publishing a manuscript that omits the reanalysis of the data which the same author performed subsequently, when 11 patients from the experimental group, for whom impedance measurements were inadequately performed, were excluded.

Additional pages can be written responding to the researcher by explaining, for example, that scans are graphically displayed to the practitioner during the course of treatment and are available for observation to Nijs group in the device still placed at the RCT site. However, from this point on, we will let the readers draw their own conclusions between the original RCT report, our scholarly response, and the researcher's response.

To conclude, from the wisdom of a great philosopher:

"I have made a ceaseless effort not to ridicule, not to bewail, not to scorn human actions, but to understand them." Baruch Spinoza -Ethics 1677

Miguel Gorenberg, MD

Bnai Zion Medical Center,

Nervomatrix Co-Founder

Haifa, Israel

E-mail: miguel.gorenberg@b-zion.org.il

Ori Kanner

CEO, Nervomatrix, Israel

Kfar Haroeh, Israel

E-mail: orik@nervomatrix.com

\section{References}

1. Aguilar Ferrandiz ME, Nijs J, Gidron Y, Roussel N, Vanderstraeten R, Van Dyck D, Huysmans E, De Kooning M5.. Autotargeted neurostimulation is not superior to placebo in chronic low back pain: $\mathrm{A}$ fourfold blind randomized clinical trial. Pain Physician 2016; 19:E707-719. 